\title{
Intra-silicone oil injection of methotrexate at the end of vitrectomy for advanced proliferative diabetic retinopathy
}

K Ghasemi Falavarjani, M Modarres,

A Hadavandkhani and A Karimi Moghaddam

\begin{abstract}
Purpose To evaluate the role of methotrexate (MTX) injected into the silicone oil at the end of pars plana vitrectomy for advanced proliferative diabetic retinopathy (PDR). Methods In this prospective comparative interventional study, eyes with severe diabetic tractional macular detachment or combined tractional/rhegmatogenous retinal detachment were included. Standard 20 gauge pars plana vitrectomy, and retinal reattachment was performed. In the case group, $250 \mu \mathrm{g}$ MTX was injected into the silicone oil at the end of surgery. The rate of retinal re-detachment associated with fibrovascular proliferation or proliferative vitreoretinopathy (PVR) was assessed. Results Overall, 38 eyes of 35 patients (19 cases and 19 controls) were studied. The two groups were matched for age, sex, preoperative visual acuity, and the type of surgery (vitrectomy alone $v s$ combined phacoemulsification/vitrectomy). Retinal re-detachment with fibrovascular proliferation or PVR occurred in seven eyes $(36.8 \%)$ in the MTX group and eight eyes $(42.1 \%)$ in the control group $(P=0.74)$. Mean change in visual acuity was $0.04 \pm 0.71$ and $0.39 \pm 0.70 \log$ MAR in the MTX and the control group, respectively $(P=0.14)$. The rate of improvement or worsening of visual acuity was similar between the two groups $(P=0.51$ and $P=0.12)$. Conclusion Intra-silicone injection of MTX at the end of vitrectomy for retinal detachment associated with severe PDR did not reduce the risk of postoperative retinal detachment due to the fibrous or fibrovascular proliferations.

Eye (2015) 29, 1199-1203; doi:10.1038/eye.2015.114; published online 10 July 2015
\end{abstract}

\section{Introduction}

The Diabetic Retinopathy Vitrectomy Study defined the benefit of pars plana vitrectomy in the management of persistent vitreous hemorrhage, and severe active fibrovascular proliferation. ${ }^{1}$ Since then, the indications for surgical management of advanced proliferative diabetic retinopathy (PDR) have steadily evolved. ${ }^{2}$ Recent tractional macular detachment (TMD) or macula threatening tractional retinal detachment (TRD), and combined tractionalrhegmatogenous retinal detachment (TRD/ RRD) are also currently considered as important indications for vitreoretinal surgery. ${ }^{2}$

Although a growing number of eyes with TMD and TRD/RRD are successfully treated with a single procedure, retinal re-detachment associated with fibrovascular proliferation or proliferative vitreoretinopathy (PVR) is still a major cause of failure of the surgery. 2,3 Previous studies have shown increased expression of inflammatory cytokines and growth factors in both PDR and PVR. ${ }^{4-6}$ Considering the major role of inflammatory mediators, it is rational to consider anti-inflammatory medications as a potential modality for prevention of proliferation of post-vitrectomy membranes.

Methotrexate (MTX) is an anti-neoplastic and anti-inflammatory agent used to treat a variety of malignancies and rheumatologic diseases. In ophthalmology, systemic and intraocular MTX has been successfully used for indeterminate uveitis, sarcoid uveitis, primary CNS lymphoma, and non-Hodgkin's lymphoma. ${ }^{78}$ Recently, small case series have reported promising results with intra-silicone injection of MTX after vitrectomy for advanced PDR. ${ }^{9,10}$ The aim of the present study was to
Department of Ophthalmology, Eye Research Center, Rassoul Akram Hospital, Iran University of Medical Sciences, Tehran, Iran

Correspondence: K Ghasemi Falavarjani, Department of Ophthalmology, Eye Research Center, Rassoul Akram Hospital, Iran University of Medical Sciences, SattarkhanNiayesh Street, Tehran 14456-13131, Iran Tel: +98 9121725850; Fax:+98 2166509162. E-mail: drghasemi@ yahoo.com

Received: 6 February 2015 Accepted in revised form: 26 May 2015 Published online: 10 July 2015

This work was presented in part orally at the Euretina Annual Meeting, London, 2014. 
evaluate the outcome of intra-silicone injection of MTX at the end of pars plana vitrectomy for eyes with TMD or TRD/RRD.

\section{Materials and methods}

In this prospective comparative interventional study, from January 2012 to January 2014, all patients who underwent pars plana vitrectomy for diabetic TMD or TRD/RRD with broad and diffuse fibrovascular membrane attachments causing underlying retinal folds were included. The surgical plane was less clearly defined in these cases and the surgical procedures were categorized as major complexity procedures. ${ }^{2}$ All patients had retinal breaks before or at the time of surgery (TRD/RRD). The case group consisted of eyes that underwent surgery from January 2013 to January 2014. In this group, intravitreal injection of MTX was performed at the end of the surgery. All eyes that underwent surgery from January 2012 to January 2013 were served as controls. In this group, no MTX injection was performed.

Patients with other intraocular diseases that may affect the course of the retinal detachment repair including uveitis, congenital vitreoretinopathies, and those with traumatic retinal detachment were excluded. Also, patients with history of previous vitreoretinal procedures were excluded. Ethics committee of the Iran University Eye Research Center approved the study. Complexity of the vitreoretinal problem, and the chance of surgical failure were explained and informed consents were obtained.

All patients received intravitreal injection of bevacizumab $(1.25 \mathrm{mg} / 0.05 \mathrm{ml})$ the night before vitrectomy as described elswhere. ${ }^{11}$ All surgeries were performed by one surgeon (KGF). Standard three-port 20 gauge pars plana vitrectomy was performed. The surgical procedure consisted of segmentation and delamination of fibrovascular membranes. Also, 'en bloc' dissection of neovascular membranes was performed when possible. No circumferential episcleral band was inserted. In cases with significant retinal shortening or unreleasable contractures, retinotomy or retinectomy was performed. Silicone oil was used for endotamponade in all eyes.

In the case group, before closure of inflow sclerotomy, $0.1 \mathrm{cc}(250 \mu \mathrm{g})$ of commercially available MTX solution (Haupt Pharma Gmbh, Wolfratshausen, Germany) was injected into the silicone-filled mid-vitreous cavity. At the conclusion of the surgery, subtenon injection of triamcinolone acetonide $(40 \mathrm{mg} / 1 \mathrm{ml})$ was performed. On the first postoperative day, topical antibiotic eye drop was administered four times daily for 2 weeks and topical betamethasone eye drop was prescribed every $2 \mathrm{~h}$ during the waking hours for 1 week and then four times daily for the next 2 weeks. The surgical procedure and postoperative medications were the same for both groups except for intra-silicone injection of MTX in the case group.

The patients were visited 1 day, 1 week, 1 month, 3 months, 6 months, and at the last follow-up after surgery. In patients without recurrent retinal detachment after surgery, the follow-up was continued for at least 6 months. Primary outcome measure was the rate of postoperative retinal detachment due to the fibrovascular proliferation or PVR, and the secondary outcome measure was the postoperative visual acuity.

Statistical analysis was performed with SPSS software (IBM Inc., Chicago, IL, USA) and $P<0.05$ was considered significant. Paired $t$-test, independent $t$-test, Fisher's exact test, and $\chi^{2}$-test were used for analysis.

\section{Results}

Overall, 38 eyes of 35 patients (19 eyes in each group) with a mean age of $51.72 \pm 7.55$ years were studied. Table 1 shows demographics of the patients. Baseline characteristics including age, sex, baseline visual acuity, and history of cataract surgery were statistically similar between the two groups. No patient developed retinal detachment during first postoperative month. Mean follow-up time was $12.3 \pm 5.3$ months.

Characteristics and outcomes of the eyes in the two groups are summarized in Table 2. Retinal re-detachment with fibrovascular proliferation or PVR occurred in seven eyes $(36.8 \%)$ in the MTX group and eight eyes $(42.1 \%)$ in the control group $(P=0.74)$, all due to the proliferation of fibrovascular or fibrous membranes. In eyes with retinal detachment, repeated vitreoretinal surgery was performed in 3 eyes in MTX group and 4 eyes in control group. Other patients rejected further surgery. Final reattachment rate was $78.9 \%$ in both groups.

Mean interval between surgery and detection of the retinal detachment in follow-up visits was $6.00 \pm 3.24$ months in the MTX group and $5.48 \pm 4.67$ months in the control group $(P=0.93)$. Silicone oil was removed from four eyes (21\%) in the MTX group and five eyes $(26.3 \%)$ in the control group $(P=1)$.

In MTX groups, the change in visual acuity after surgery was not statistically significant $(P=0.77)$; however, statistically significant improvement was found in the control group $(P=0.02)$. Mean change in visual acuity was $0.04 \pm 0.71$ and $0.39 \pm 0.70 \mathrm{LogMAR}$ in MTX and control group, respectively $(P=0.14)$. Visual acuity improved in seven eyes $(36.8 \%)$ in the MTX group and nine eyes $(47.3 \%)$ in controls $(P=0.51)$. A decrease in visual acuity was found in seven eyes $(36.8 \%)$ in the MTX group and two eyes $(10.5 \%)$ in the control group $(P=0.12)$. 


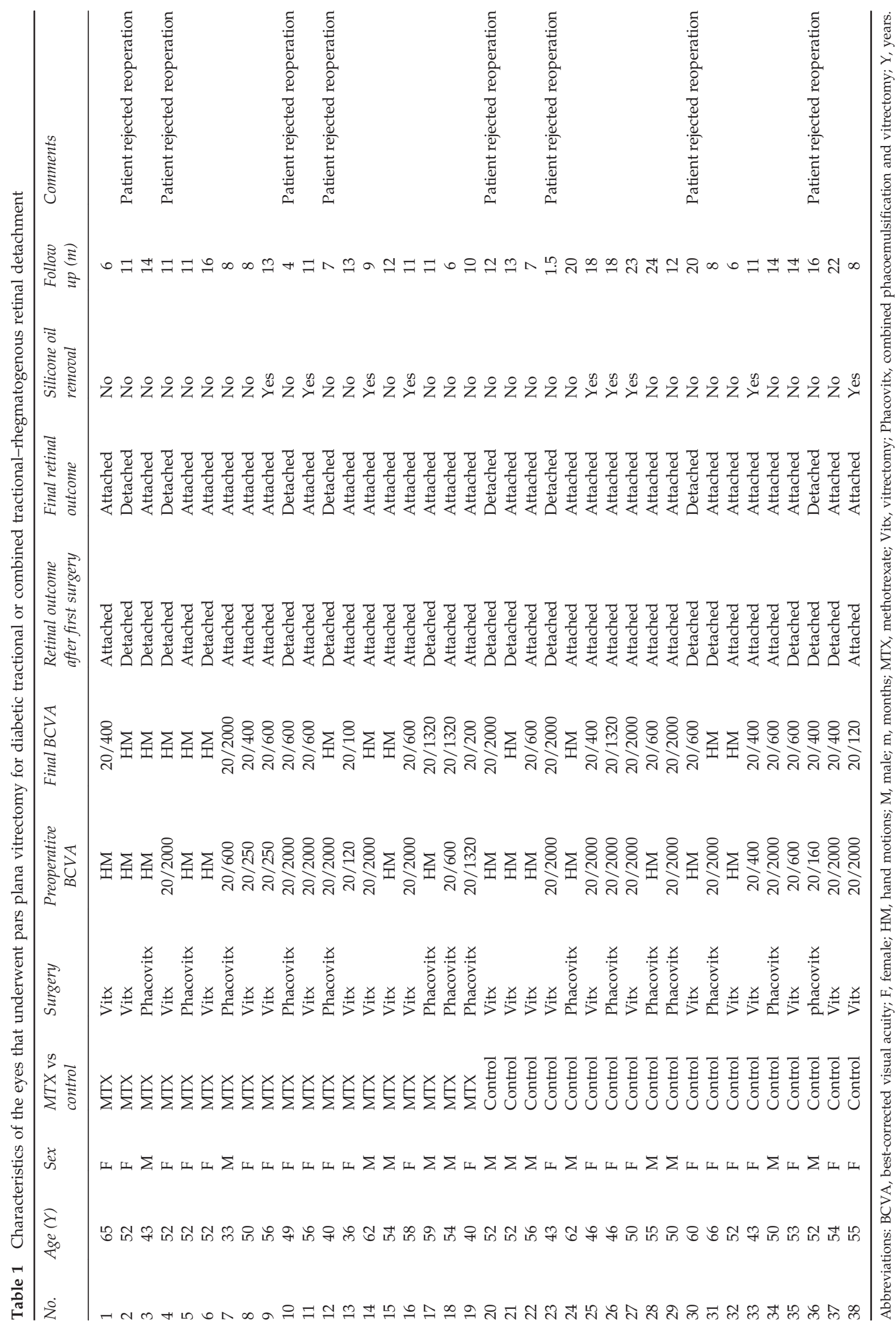


Table 2 Baseline characteristics and outcomes of diabetic patients with tractional or combined tractional-rhegmatogenous retinal detachment who underwent pars plana vitrectomy in the methotrexate and control group

\begin{tabular}{|c|c|c|c|}
\hline & MTX group & Control group & P-value \\
\hline Number of eyes & 19 & 19 & \\
\hline Age (year; mean $\pm \mathrm{SD}$ ) & $50.61 \pm 8.93$ & $52.83 \pm 5.92$ & $0.38^{\mathrm{a}}$ \\
\hline Sex (male/female) & $6 / 12$ & $8 / 10$ & $0.49^{\mathrm{b}}$ \\
\hline Lens status (phakic/pseudophakic) & $17 / 2$ & $16 / 3$ & $1^{\mathrm{c}}$ \\
\hline Surgery (vitrectomy/combined phacoemulsification and vitrectomy) & $11 / 8$ & $12 / 7$ & $0.74^{\mathrm{b}}$ \\
\hline Baseline visual acuity $(\log M A R)($ mean $\pm S D)$ & $2.14 \pm 0.76$ & $2.24 \pm 0.65$ & $0.23^{\mathrm{a}}$ \\
\hline Final visual acuity $(\operatorname{logMAR} ;$ mean $\pm S D)$ & $2.09 \pm 0.84$ & $1.85 \pm 0.68$ & $0.33^{\mathrm{a}}$ \\
\hline Change in visual acuity (logMAR; mean $\pm S D)$ & $0.04 \pm 0.71$ & $0.39 \pm 0.70$ & $0.14^{\mathrm{a}}$ \\
\hline Number of eyes with retinal re-detachment after first vitrectomy & 7 & 8 & $0.74^{\mathrm{b}}$ \\
\hline
\end{tabular}

Abbreviation: MTX, methotrexate. ${ }^{\mathrm{a}}$ t-test. ${ }^{\mathrm{b}} \chi^{2}$-test. ${ }^{\mathrm{c}}$ Fisher's exact test.

\section{Discussion}

Although many angiogenic and cytokine mediators are involved in the development and progression of diabetic retinopathy, inflammatory reaction seems to have a critical role in the pathophysiology of the diabetic retinopathy. ${ }^{4}$ Previous studies have shown a significant increase in the level of inflammatory mediators such as ICAM-1, VCAM-1, IL-6, IL-8, IL-10, and TNF- $\alpha$ in PDR. ${ }^{4}$ Considering the prominent role of inflammation in pathogenesis of fibrovascular proliferation and PVR, which are the known complications after diabetic vitrectomy, the use of anti-inflammatory medications has been suggested. Previous studies have reported contradictory results after intravitreal injection of triamcinolone acetonide in patients undergoing vitrectomy for PDR. ${ }^{12,13}$ Jonas et al ${ }^{12}$ reported a reattachment rate of $89.7 \%$ after intravitreal injection of $15-20 \mathrm{mg}$ of crystalline triamcinolone acetonide at the end of surgery in a series of patients with advanced PDR. However, in a comparative study, the same group reported similar outcomes between the intravitreal injection of triamcinolone acetonide and the control group. ${ }^{13}$ Moreover, intravitreal injection of steroids may result in cataract formation and steroid-induced glaucoma.

MTX is a folate analog and works by inhibiting dihydrofolate reductase, which is involved in the synthesis of thymidylate and purine nucleotide, resulting in the inhibition of rapidly dividing cells such as lymphocytes. Although MTX was first introduced as an antiproliferative agent, it is now clear that many of the anti-inflammatory effects of MTX are mediated by adenosine. ${ }^{14}$ Several studies have shown that MTX modulates the humoral and cellular responses and suppresses the level and activity of TNF and various ILs. ${ }^{14,15}$ Previous studies have reported potent antiinflammatory properties of the intravitreal MTX in patients with noninfectious uveitis. ${ }^{16,17}$ Moreover, intravitreal injection of MTX has less complication compared with the triamcinolone acetonide, another potent widely used anti-inflammatory agent. ${ }^{9}$ Two studies reported the use of intra-silicone MTX for surgical indications other than intraocular lymphoma. ${ }^{9,10}$ In these reports, a total of seven eyes had vitrectomy for diabetic TRD and TRD/RRD. Visual acuity improved in two eyes, remained stable in three eyes and decreased in others. Anatomical outcome was reported for three eyes and the retina was attached at the end of follow-up in these eyes. On the whole, the available literature has defined a positive role for MTX in controlling proliferative conditions, including inflammatory response such as postoperative inflammation. However, our study did not show a beneficial result from its injection at the end of vitrectomy for prevention of glial proliferation and the resultant retinal detachment in PDR. This may be ascribed to several reasons. The sample size was small and may not have had enough power to detect the difference between the two groups. Also, therapeutic half-life of MTX after a single intravitreal injection is only 3-5 days, 18,19 whereas the process of glial proliferation after vitrectomy has a protracted course and may take several weeks to months. It is possible that multiple postoperative injections of MTX over few weeks would prevent membrane formation more efficiently. Another possibility is that the slow diffusion of MTX out of the silicone oil may make its available concentration in the perisilicone space inadequate for an efficient therapeutic effect.

Previous studies have shown that intravitreal injection of $400 \mu \mathrm{g}$ of MTX is nontoxic for the retina. ${ }^{8}$ Also, the safety of intra-silicone injection of 200 and $400 \mu \mathrm{g}$ doses of MTX has been previously tested. ${ }^{9,10}$ Considering the reduced clearance in vitrectomized eyes filled with silicone oil, we selected the lower dose to allay the concerns of toxicity. ${ }^{10}$ Higher doses of MTX may have better effect while being still in the safe range. ${ }^{10}$

Our study has some limitations. The non-randomized design of study is one the major limitations. The study is underpowered to detect small differences between the two groups. Based on our current results, the sample size needed for a statistical power of $80 \%$ to detect a $10 \%$ 
increase in anatomical success is 268 eyes in each group and recruitment of such a large number of patients may not be feasible. We did not include eyes with other complications of PDR such as non-clearing vitreous hemorrhage. Additional studies with larger number of patients, and possibly using multiple injections or higher doses are needed to further elucidate the effects of MTX as an adjunct in the surgical management of PDR.

\section{Summary}

What was known before

- Retinal re-detachment associated with fibrovascular proliferation or proliferative vitreoretinopathy (PVR) is a major cause of failure of the surgery for advanced proliferative diabetic retinopathy (PDR).

- Inflammatory cytokines and growth factors increase in both PVR and PDR.

What this study adds

- Intra-silicone injection of methotrexate at the end of vitrectomy for retinal detachment associated with severe proliferative diabetic retinopathy did not reduce the risk of postoperative retinal detachment due to the fibrous or fibrovascular proliferations.

\section{Conflict of interest}

The authors declare no conflict of interest.

\section{References}

1 Early vitrectomy for severe proliferative diabetic retinopathy in eyes with useful vision. Clinical application of results of a randomized trial-Diabetic Retinopathy Vitrectomy Study Report 4. The Diabetic Retinopathy Vitrectomy Study Research Group. Ophthalmology 1988; 95: 1321-1334.

2 Newman DK. Surgical management of the late complications of proliferative diabetic retinopathy. Eye (Lond) 2010; 24(3): 441-449.

3 Meleth AD, Carvounis PE. Outcomes of vitrectomy for tractional retinal detachment in diabetic retinopathy. Int Ophthalmol Clin 2014; 54(2): 127-139.

4 Gologorsky D, Thanos A, Vavvas D. Therapeutic interventions against inflammatory and angiogenic mediators in proliferative diabetic retinopathy. Mediat Inflamm 2012; 2012: 629452.
5 Zhou J, Wang S, Xia X. Role of intravitreal inflammatory cytokines and angiogenic factors in proliferative diabetic retinopathy. Curr Eye Res 2012; 37(5): 416-420.

6 Ricker LJ, Kijlstra A, Kessels AG, de Jager W, Liem AT, Hendrikse $\mathrm{F}$ et al. Interleukin and growth factor levels in subretinal fluid in rhegmatogenous retinal detachment: a case-control study. PLoS One 2011; 6(4): e19141.

7 Samson CM, Waheed N, Baltatzis S, Foster CS. Methotrexate therapy for chronic noninfectious uveitis: analysis of a case series of 160 patients. Ophthalmology 2001; 108: 1134-1139.

8 Smith JR, Rosenbaum JT, Wilson DJ, Doolittle ND, Siegal T, Neuwelt EA et al. Role of intravitreal methotrexate in the management of primary central nervous system lymphoma with ocular involvement. Ophthalmology 2002; 109: 1709-1716.

9 Hardwig PW, Pulido JS, Bakri SJ. The safety of intraocular methotrexate in silicone-filled eyes. Retina 2008; 28(8): 1082-1086.

10 Hardwig PW, Pulido JS, Erie JC, Baratz KH, Buettner H. Intraocular methotrexate in ocular diseases other than primary central nervous system lymphoma. Am J Ophthalmol 2006; 142(5): 883-885.

11 Modarres M, Nazari H, Falavarjani KG, Naseripour M, Hashemi M, Parvaresh MM. Intravitreal injection of bevacizumab before vitrectomy for proliferative diabetic retinopathy. Eur J Ophthalmol 2009; 19(5): 848-852.

12 Jonas JB, Hayler JK, Söfker A, Panda-Jonas S. Intravitreal injection of crystalline cortisone as adjunctive treatment of proliferative diabetic retinopathy. Am J Ophthalmol 2001; 131(4): 468-471.

13 Jonas JB, Söfker A, Degenring R. Intravitreal triamcinolone acetonide as an additional tool in pars plana vitrectomy for proliferative diabetic retinopathy. Eur J Ophthalmol 2003; 13(5): 468-473.

14 Chan ES, Cronstein BN. Molecular action of methotrexate in inflammatory diseases. Arthritis Res 2002; 4(4): 266-273.

15 Cronstein B. How does methotrexate suppress inflammation? Clin Exp Rheumatol 2010; 28(5 Suppl 61): S21-S23.

16 Taylor SR, Habot-Wilner Z, Pacheco P, Lightman SL. Intraocular methotrexate in the treatment of uveitis and uveitic cystoid macular edema. Ophthalmology 2009; 116(4): 797-801.

17 Taylor SR, Banker A, Schlaen A, Couto C, Matthe E, Joshi L et al. Intraocular methotrexate can induce extended remission in some patients in noninfectious uveitis. Retina 2013; 33(10): 2149-2154

18 Fishburne BC, Wilson DJ, Rosenbaum JT, Neuwelt EA. Intravitreal methotrexate as an adjunctive treatment of intraocular lymphoma. Arch Ophthalmol 1997; 115: $1152-1156$.

19 de Smet MD, Vancs VS, Kohler D, Solomon D, Chan CC. Intravitreal chemotherapy for the treatment of recurrent intraocular lymphoma. Br J Ophthalmol 1999; 83: 448-451. 